oat-cell carcinoma. ${ }^{4}$ The evidence we have presented here suggests that in the testicular tumours ${ }^{67} \mathrm{Ga}$ may be more consistently taken up by seminoma than by teratoma. In patients who are suffering from what it presumed to be a pure seminoma the change from a positive scan to a negative scan together with a change in the clinical course of the disease suggests that a teratoma may have come to dominate the clinical picture.

Pinsky et $a l^{5}$ have recorded the largest series of ${ }^{67} \mathrm{Ga}$-citrate scans in patients with testicular tumours. Only abdominal scanning was performed, but this proved useful in assessing the spread of tumour to the abdominal lymph nodes. Embryonal carcinomas (anaplastic teratomas) tended to give positive scans, but there were no patients in the series who had disseminated seminomas and few who had more differentiated teratomas.

It is not known why seminomas, like lymphomas, have a high affinity for ${ }^{67} \mathrm{Ga}$. Swarzendruber et al ${ }^{6}$ performed electron microscopic autoradiography on thymus and lymph node tissue of leukaemic mice and found that ${ }^{67} \mathrm{Ga}$ activity was predominantly in "lysosomal-like" granules within tissue macrophages. Inflammatory cells are known to have a high level of lysosomal activity. Seminomas are tumours characterised clinically by a good response to treatment and often histologically by the presence of ganulomata and lymphocytic infiltration, whereas teratoma of the testis is not generally characterised by either of these features. These characteristics may contribute to the particularly good imaging properties of seminoma.

Case 1 illustrates how useful this technique is in following up patients with seminoma of the testis. The deposit of seminoma below the right lobe of the liver was noted six months before his clinical condition demanded exploratory laparotomy; the gallium scan was the only test that detected the tumour recurrence.
Case 2 illustrates a difficult problem in managing patients with seminoma of the testis. When a patient who has been diagnosed as having a pure seminoma then develops a recurrence in a previously treated area or responds poorly to standard treatment careful consideration is needed about whether a teratoma has developed. In case 2 a tumour that had taken up ${ }^{67} \mathrm{Ga}$ no longer did so, which suggested that the character of the tumour had changed. Case 3 illustrates the value of gallium scanning in the initial staging of a patient and in monitoring the response to treatment.

In conclusion, we have found this technique to be of great value in diagnosing seminoma of the testis and following up patients. The apparent difference in imaging characteristics between seminoma and teratoma requires confirmation and if proved may have some value in the differential diagnosis of patients with combined tumour of the testis.

We thank Mr D Manning, superintendent radiographer, and his staff for performing the scans in this study.

\author{
References \\ ${ }^{1}$ Lomas, F, Dibos, P E, and Wagner, H N, New England fournal of Medicine, \\ 1972, 286, 1323. \\ ${ }^{2}$ Milder, M S, et al, Cancer, 1973, 6, 1350. \\ ${ }^{3}$ Collins, D H, and Pugh, R C, British.Fournal of Urology, 1964, 36, suppl \\ I-II. \\ 4 van der Schoot, J B, Groen, A S, and de Jong, J, Thorax, 1972, 27, 543. \\ 5 Pinsky, S M, et al, fournal of Nuclear Medicine, 1973, 14, 339. \\ Swartzendruber, D C, Nelson, B, and Hayes, R L, Fournal of the National \\ Cancer Institute, 1972, 46, 941.
}

\title{
Controlled trial of oxygen in uncomplicated myocardial infarction
}

\author{
J M RAWLES, A C F KENMURE
}

evidence of benefit from the routine administration of oxygen in uncomplicated myocardial infarction.

\section{Summary}

Two-hundred consecutive patients thought to have suffered a myocardial infarction were admitted to a randomised, double-blind controlled trial of oxygen or air administered by MC mask throughout the first 24 hours in hospital. Forty-three patients in whom myocardial infarction was not subsequently confirmed were excluded from the analysis. The remaining air and oxygen groups were comparable except for a significantly higher $\mathrm{PaO}_{2}$ and serum aspartate aminotransferase level in the oxygen group. There was no significant difference in mortality, incidence of arrhythmias, use of analgesics, or systolic time intervals between the two groups, although a higher incidence of sinus tachycardia was found in those given oxygen. There appears to be no

Coronary Care Unit, Aberdeen Royal Infirmary, Aberdeen AB9 2ZB

J M RAWLES, BSC, MRCP, consultant physician (present address: West Cornwall Hospital, Penzance TR18 2PF)

A C F KENMURE, MD, FRCPGLAS, consultant cardiologist

\section{Introduction}

The use of oxygen in "severe angina pectoris" was first described in 1900 by Steele. ${ }^{1}$ Clinical improvement after oxygen inhalation in four patients with acute myocardial infarction was reported by Levy and Barach in $1930 .^{2}$ Since then the use of oxygen in myocardial infarction has been advised in most standard medical texts. ${ }^{3}+$ The discovery that patients with myocardial infarction usually have a degree of arterial hypoxaemia that may be corrected with oxygen has lent support to this advice. ${ }^{5}$ It has been suggested that increasing the arterial oxygen tension may reduce the area of ischaemia around an infarct ${ }^{6}$ or reduce the incidence of arrhythmias, and such effects have been shown in animals after coronary artery ligation. ${ }^{7}$ Early workers also reported that oxygen reduced the pain of myocardial infarction. ${ }^{8} 9$ Despite these various claims the efficacy of oxygen in myocardial infarction has received remarkably little attention, although the haemodynamic and metabolic effects have been studied. Only two controlled trials of oxygen treatment have been carried out, and these were under hyperbaric conditions; the results were inconclusive, although neither trial showed a significant reduction in mortality. ${ }^{1011} \mathrm{~A}$ study to determine the efficacy of oxygen 
is thus long overdue, ${ }^{12}$ and we report here what we believe to be the first controlled trial of the effect of oxygen treatment at atmospheric pressure on the severity of infarction, the incidence of arrhythmias, and the use of analgesics.

\section{Patients and methods}

The trial was conducted in this coronary care unit, where admissions are restricted to patients aged under 65 . Two-hundred consecutive patients thought by the admitting medical officer to have had a myocardial infarction in the preceding 24 hours were admitted to the trial. Patients were excluded if they had clinical evidence of right or left heart failure, chronic bronchitis or emphysema or breathlessness from any other cause, or if they had been transferred from other wards for the treatment of arrhythmias or had undergone a cardiac arrest before admission or suffered from cardiogenic shock. After the decision was made to admit a patient to the trial the unit sister opened a numbered sealed envelope containing the randomised allocation of the patient to oxygen or air inhalation. Either oxygen or compressed air was administered by means of an MC mask at a flow rate of $61 / \mathrm{min}$ for 24 hours. The cylinders were shrouded so that the identity of the gas was not apparent to the medical staff or patients. If during the first 24 hours it became mandatory to give oxygen because of cardiac arrest or developing left ventricular failure, then the mask was disconnected from the cylinder and connected to the piped wall supply of oxygen without disclosing to the medical staff the identity of the former gas. In the analysis of the results such patients were included in the group to which they were first allocated.

Myocardial infarction was confirmed when any two of the following three conditions were present: a classical history of gripping pain across the chest unaffected by position, respiration, rest, or glyceryl trinitrate and lasting more than half an hour; a rise of the serum aspartate aminotransferase level above $20 \mathrm{IU} / \mathrm{ml}$; an abnormal electrocardiogram with sequential ST and $\mathrm{T}$-wave changes with or without pathological $\mathrm{Q}$ waves. When myocardial infarction was not confirmed treatment and investigation in the first 24 hours continued unchanged but the results were excluded from the final analysis.

Every trial patient had an electrocardiogram recorded for two minutes every hour, and the heart rate, presence of arrhythmias, and number and types of ectopic beats were noted. This constituted a 1 in 30 sample of the first 24 hours of monitoring. Arrhythmias occurring at other times were also recorded, and their treatment with drugs or cardioversion was unaffected by the trial. Pain was treated with diamorphine in a standard 5-mg dose intramuscularly as required and a retrospective record made of the number of doses given to each patient. At the time of the trial the nursing staff were unaware that the use of analgesics was being assessed. In 50 patients the $\mathrm{PaO}_{2}$ was measured during inhalation treatment but the result was not disclosed until the assessment of the patient's progress and complications was complete. In 17 patients systolic time intervals were measured ${ }^{13}$ at about noon during the first 24 hours after admission while on oxygen or air and 24 hours later when breathing room air. The maximum serum aspartate aminotransferase levels were recorded. Results were analysed by using the $\chi^{2}$ test with Yates's correction for comparison of frequencies, and Student's $t$ test for comparison of mean values. ${ }^{14}$

In view of the absence of published information on the efficacy of oxygen treatment or even of a commonly held clinical impression that oxygen is beneficial, and since many patients with myocardial infarction are treated at home without oxygen and without obvious detriment, we felt it ethically justifiable to withhold oxygen from the air group.

\section{Results}

Ninety-five patients were allocated to the air group and 105 to the oxygen group. Myocardial infarction was not confirmed in 18 patients in the air group and 25 in the oxygen group, and these patients were therefore excluded from subsequent analysis. These proportions are not significantly different, and details of the patients are given in table I. As expected, the $\mathrm{PaO}_{2}$ of the withdrawn patients given oxygen exceeded that of the withdrawn patients given air, but there were no other important differences between the groups. Details of the remaining 157 patients are given in table II. The oxygen and air groups were comparable in mean age and sex distribution, and, as expected, the oxygen group had a higher $\mathrm{PaO}_{2}$. The mean serum aspartate aminotransferase level was significantly higher in the oxygen group than in the air group $(P<0.05)$, and there were nine deaths in the
TABLE I-Details of patients in whom myocardial infarction was not confirmed. Mean values are given $\pm S E$ of mean

\begin{tabular}{|c|c|c|c|c|}
\hline & & Air group & Oxygen group & $\mathbf{P}$ \\
\hline 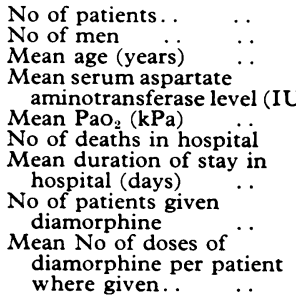 & $\begin{array}{c}\cdots \\
\cdots \\
\cdots \\
\mathrm{J} / \mathrm{ml}) \\
\cdots \\
\cdots \\
\cdots \\
\cdots\end{array}$ & $\begin{array}{c}18 \\
17 \\
50 \cdot 8=2 \cdot 4 \\
18 \cdot 3 \pm 3 \cdot 0 \\
11 \cdot 2 \pm 0 \cdot 17(2) \\
0 \\
9.9 \pm 1.6 \\
3\end{array}$ & $\begin{array}{c}25 \\
19 \\
51 \cdot 3 \pm 1 \cdot 7 \\
15 \cdot 8 \pm 1 \cdot 1 \\
23.7 \pm 1 \cdot 32(12) \\
0 \\
11 \cdot 1 \pm 1 \cdot 3 \\
11\end{array}$ & $\begin{array}{l}\text { NS } \\
\text { NS } \\
\text { NS } \\
\text { NS } \\
<0 \cdot 001 \\
\text { NS } \\
\text { NS } \\
\text { NS }\end{array}$ \\
\hline
\end{tabular}

Figures in parentheses indicate numbers of patients where these differ from total in group. $=$ Not significant.

Conversion: SI to traditional units- $\mathrm{PaO}_{2}: 1 \mathrm{kPa} \approx 7.5 \mathrm{~mm} \mathrm{Hg}$.

TABLE II-Details of patients with definite myocardial infarction. Mean values are given $\pm S E$ of mean

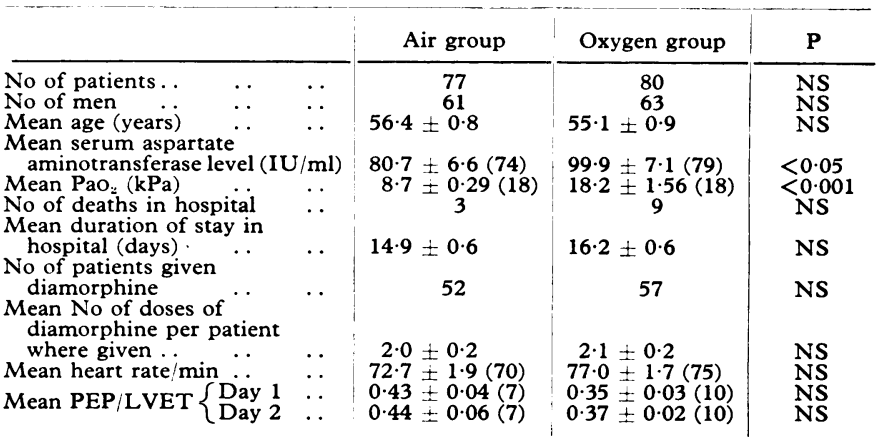

Figures in parentheses indicate numbers of patients where these differ from total in PEP/LVET $=$ Pre-ejection phase/left ventricular ejection time.

TABLE III-Numbers of patients with various arrhythmias after myocardial infarction

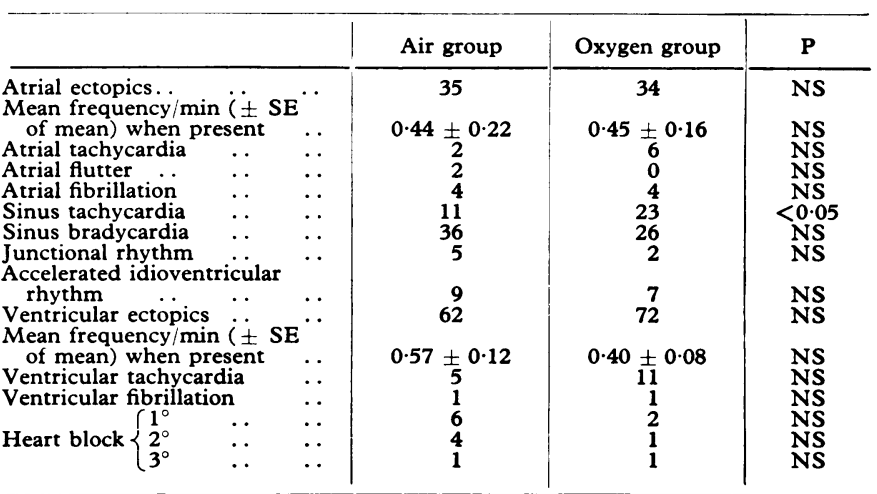

oxygen group compared with three in the air group, but this difference was not significant. One death in the oxygen group and two deaths in the air group occurred while the patients were on oxygen or air. No other significant differences between the two groups were found.

Systolic ejection times did not differ between the two groups on the first or second day, nor was there a significant difference in paired recordings in the same patients between the first and second days. Table III gives the incidence of arrhythmias in the two groups, the only significant difference being a more frequent occurrence of sinus tachycardia in those given oxygen $(P<0.05)$.

\section{Discussion}

The rationale for the routine use of oxygen in acute myocardial infarction is based on two concepts. In the first place, arterial 
hypoxaemia is present in many patients after myocardial infarction and it has been shown experimentally that hypoxia may predispose to ventricular fibrillation. ${ }^{15}$ It is hoped, therefore, that oxygen administration by correcting the arterial hypoxia might reduce the incidence of ventricular fibrillation and other serious arrhythmias. Secondly, it has been suggested that by increasing the amount and tension of oxygen in the arterial blood oxygen treatment might allow improved oxygenation to the peripheral zone of the infarcted area of myocardium and thus reduce the final size of the infarct. ${ }^{6}$

We found no evidence that oxygen given to patients with uncomplicated myocardial infarction is beneficial in either of these respects. In particular, it did not appear to reduce the incidence of minor or major arrhythmias, and, indeed, the incidence of sinus tachycardia was greater when receiving oxygen. There was no improvement in left ventricular function as determined clinically or by measurement of systolic time intervals. Moreover, mortality was unaffected whether oxygen or air was breathed. On the other hand, a higher mean level of serum aspartate aminotransferase was found in those given oxygen. While this might be accounted for by chance allocation of patients with more extensive infarction to the oxygen group, or to the timing of blood sampling for biochemical examination, there is less than a $5 \%$ probability that this occurred. Alternatively, possibly oxygen treatment, far from being beneficial, actually produced a deleterious effect.

In normal people oxygen inhalation causes a reduction of cardiac output, largely due to a fall in heart rate, and a rise in systemic blood pressure. ${ }^{16}$ Hence there is an increase in systemic vascular resistance that is due to a direct vasoconstrictive effect of oxygen. In patients with myocardial infarction the haemodynamic effects of oxygen administration are variable but broadly similar to those in normal people, a reduction of cardiac output occurring with maintenance of blood pressure. The heart rate is generally unchanged, but, again, a rise in the peripheral resistance occurs, although left ventricular work is not increased. It has therefore been suggested that on balance the haemodynamic effects of oxygen in myocardial infarction are beneficial, the rise in arterial pressure outweighing the fall in cardiac output. ${ }^{17}$

The metabolic changes that accompany oxygen administration have also been studied. ${ }^{517}$ The arterial hypoxia commonly present in patients with myocardial infarction is usually eliminated by oxygen inhalation. Raised arterial lactate concentrations are also often found, and after treatment with oxygen in high concentrations these levels fall, suggesting that oxygen delivery to tissues is generally improved. In patients with ischaemic heart disease, however, abnormally raised coronary venous lactate levels may be produced or accentuated by inhalation of $100 \%$ oxygen. ${ }^{18}$ It appears, therefore, that, despite overall improvement in oxygenation, oxygen delivery to individual organs is not necessarily increased. Cerebral, ${ }^{19}$ renal, ${ }^{20}$ and retinal ${ }^{21}$ vasoconstriction has been reported and coronary blood flow is also reduced after inhalation of high concentrations of oxygen. ${ }^{22}$ The decrease in myocardial blood flow prevents any additional oxygen delivery to the heart despite a considerable increase in arterial oxygen content; indeed, the decrease in blood flow may be so great as to reduce the total amount of oxygen available to the heart. Thus oxygen treatment far from achieving the desired effect of limiting the ischaemic area might actually result in an extension of the area of infarction, and this could offer one explanation for the increased aspartate levels found in the oxygen group in this study.

In conclusion, therefore, although we have obtained some suggestive evidence of a deleterious effect of oxygen, the findings are tentative. What has been shown is that the administration of oxygen does not appear to be of any benefit to patients with uncomplicated myocardial infarction. This does not mean, of course, that oxygen should be withheld from patients with obvious hypoxia, as in the presence of severe left ventricular failure, but there seems to be little place for routine oxygen administration to all patients with acute myocardial infarction.

\section{References}

${ }^{1}$ Steele, C, British Medical fournal, 1900, 2, 1568

${ }^{2}$ Levy, R L, and Barach, A L, fournal of the American Medical Association, 1930, 94, 1363

${ }^{3}$ Harrison, T R, Harrison's Principles of Internal Medicine, 7th edn, p 1203. Tokyo, McGraw-Hill Kogakusha, 1974.

${ }^{4}$ Beeson, P B, and McDermott, W (editors), in Cecil-Loeb Textbook of Medicine, 13th edn, p 1036. Philadelphia, Saunders, 1971.

5 MacKenzie, G J, et al, Lancet, 1964, 2, 825.

${ }^{6}$ Sayen, J J, et al, fournal of Clinical Investigation, 1951, 30, 932.

7 Smith, G, and Lawson, D D, Scottish Medical fournal, 1958, 3, 346

${ }^{8}$ Rizer, R I, Minnesota Medicine, 1929, 12, 506.

9 Boland, E W, Fournal of the American Medical Association, 1940, 114, 1512.

10 Cameron, A J V, et al, in Hyperbaric Oxygenation, p 277. Edinburgh and London, Livingstone, 1965.

11 Thurston, J G B, et al, Quarterly fournal of Medicine, 1973, 42, 751.

12 Saltzman, H A, Circulation, 1975, 52, 357.

13 Weissler, A M, Harris, W S, and Schoenfeld, C D, American fournal of Cardiology, 1969, 23, 577.

14 Fisher, R A, Statistical Methods for Research Workers, 12th edn. Edinburgh, Oliver and Boyd, 1954.

15 Burn, J H, and Hukovic, S, British fournal of Pharmacology, 1960, 15, 67.

${ }_{16}$ Kenmure, A C F, et al, Fournal of Applied Physiology, 1972, 32, 223.

17 Kenmure, A C F, et al, British Medical fournal, 1958, 4, 360.

18 Bourassa, M G, et al, American fournal of Cardiology, 1969, 24, 172.

19 Kety, S S, and Schmidt, C F, fournal of Clinical Investigation, 1948, 27, 484.

20 Aber, G M, Harris, A M, and Bishop, J M, Clinical Science, 1964, 26, 133.

21 Dollery, C T, et al, Lancet, 1964, 2, 291.

${ }^{22}$ Kenmure, A C F, et al, Cardiovascular Research, 1971, 5, 483.

\section{Patients, methods, and results}

Cultures for yeasts were taken from the coronal sulcus and fossa navicularis of 205 new patients attending the Whitechapel Clinic. None was diabetic,

and already known contacts of women with yeast infections were excluded. Gram-stained smears of material from the two sites were also examined for spores and mycelium, but as the first 100 specimens from the fossa navicularis were all negative only the coronal sulcus was subsequently examined. Primary isolation was on Sabouraud dextrose agar and candida (Oxoid) plates. Candida albicans was identified by germ tube and chlamydospore formation. Several of the other yeasts recovered were kindly identified by the Mycological Reference Laboratory.

Thirty of the 205 men had balanitis ( 12 were yeast-positive-all $C$ albicans) and to avoid bias were excluded from the analysis. Of the 175 men without balanitis $32(18 \%)$ had evidence of yeast infection. Twenty-four $(14 \%)$ had positive cultures, smears also being positive in six of these (mycelium seen in one), and eight had positive smears alone (none had mycelium). Only one of the 32 had received an antibiotic in the previous three months. Candida species were isolated in $17(10 \%) ; 10(6 \%)$ were $C$ albicans, two $C$ paropsilosis, and five were unidentified but shown not to be $C$ albicans. Torulopsis candida was isolated in three cases, making a total of $20(11 \%)$ isolations of yeast species which might be potential causes of vaginitis. The sites of recovery. are shown in the table. The remaining four positive cultures were of Trichosporon cutaneum. 